\title{
VERBUNDTAG 2015, NEUE OBVSG-WEBSITE, REORGANISA- TION DER ZDB-BEARBEITUNG IM OBV: MITTEILUNGEN DER OBVSG
}

von Peter Klien

Vier Linzer Augen sehen mehr als zwei Verbundtag 2015 an der Johannes Kepler Universität in Linz

Heuer zum elften Mal veranstaltete der Österreichische Bibliothekenverbund seinen jährlichen Verbundtag. Diesmal lud die Johannes Kepler Universität Linz auf ihren schönen Campus. Rund 100 Expertinnen und Experten trafen sich in offener Atmosphäre, um über Entwicklungen im Bibliothekswesen, anstehende Aufgaben des Verbundes und mögliche zukünftige Strategien zu beraten.

Das Vormittagsplenum stand unter dem Generalthema: „Neue Infrastrukturen in Österreichs Wissenschaft". Barbara Sánchez Solís und Raman Ganguly (Universität Wien) erläuterten „e-Infrastructures Austria“, das Projekt für den koordinierten Aufbau von nationalen Repositorieninfrastrukturen in Forschung und Lehre. Danach stellte Bruno Bauer (Meduni Wien) die Frage: „Open Access in Österreich - eine Aufgabe (auch) für den Österreichischen Bibliothekenverbund?"

Am Nachmittag warteten zahlreiche parallele Workshops. Dabei standen folgende Themen zur Auswahl: RDA (Implementierung in Aleph, Tests und Schulung im OBV), Visual Library (Neues zum konsortialen Dienst der OBVSG), E-Books im Österreichischen Bibliothekenverbund, Provenienzerschließung im OBV, ein Follow-Up zum letztjährigen Schwerpunkt Datenkorrekturen sowie Informationen zum Relaunch der OBVSG-Homepage.

Geselligen Ausklang fand der Verbundtag bei einer Führung durch das LENTOS Kunstmuseum und einem gemeinsamen Abendessen am Donauufer.

\section{Homepage neu macht der Mai \\ Der Relaunch der OBVSG-Website}

Die OBVSG hat das ausständige Upgrade ihres Content Management Systems (CMS) zum Anlass genommen, die gesamte Website einem ausgiebigen Relaunch zu unterwerfen. 
Seit Juni 2006 kommt für die Homepage der OBVSG das Open Source CMS TYPO3 zum Einsatz. Dessen neue Version 6 stellt einen harten Bruch mit den Vorgängerversionen dar, weil Grafik-Design (die so genannten Templates) und viele Funktionalitäten (die so genannten Extensions) komplett neu adaptiert werden müssen. Eine kurze Überprüfung, ob man unter diesen Umständen überhaupt mit TYPO3 weitermachen wollte, hat aus mehreren Gründen bald zu einem positiven Ergebnis geführt. So ist TYPO3 inzwischen zu einer Art Standard für Webseiten im akademischen Bereich geworden. Die Komplexität der OBVSG-Homepage sowie die weiterhin sehr große Entwicklergemeinde sprachen ebenfalls für die Beibehaltung der Software.

Bei der Implementierung der neuen Version wollte man allerdings aktuellen Webtrends Beachtung schenken. Daher werden nun dieselben Inhalte auf Smartphone, Tablet, Laptop oder Desktop in Hoch- oder Querformat in zwar verschiedener Aufmachung, immer aber in gleich guter Qualität präsentiert (so genanntes responsives Design).

Umgesetzt wurde das aufwändige Projekt über einen Zeitraum von sechs Monaten in Kooperation mit lang jährigen OBVSG-Partnern in den Bereichen Grafik, Entwicklung und TYPO3.

\section{Kollektive Weisheit}

\section{Zur Reorganisation der ZDB-Bearbeitung im OBV}

Die Zeitschriftendatenbank (ZDB) ist die zentrale bibliografische Datenbank für Titel- und Besitznachweise fortlaufender Sammelwerke in Deutschland und Österreich, zum Beispiel von Fachzeitschriften, Zeitschriften und Zeitungen.

Sie wird zentral an der Deutschen Nationalbibliothek vorgehalten (Datenbank und Systempflege) und ist im Internet frei nutzbar. Die Zeitschriftentitel österreichischer Bibliotheken wurden bis 2012 über die Österreichische Nationalbibliothek in die ZDB eingebracht. Danach war im OBV aus Ressourcengründen keine voll institutionalisierte (i.e. an einer Stelle konzentrierte) Zentralredaktion mehr möglich. Stattdessen wurde ein kooperatives Redaktionskollektiv aufgebaut, in dem neben der ÖNB acht Universitätsbibliotheken aus ganz Österreich vertreten sind. Eine künftige Erweiterung des Eingeberkreises ist wünschenswert, die Teilnahme möglichst vieler Bibliotheken wird angestrebt. Ziel ist außerdem die Etablierung einer zuständigen Regionalkompetenzstelle pro Bundesland. 
Aus technischer Sicht hervorzuheben ist, dass die ZDB eine Aleph-fremde Umgebung darstellt. Der schreibende Zugriff auf die Daten erfordert daher die Installation zusätzlicher Software. Daneben muss das ZDBspezifische Eingabeformat erlernt werden. Alle Institutionen des Redaktionskollektivs haben sich für diesen Weg entschieden. Alternative ist die Eingabe über eine Web-Schnittstelle, die allerdings mit Beschränkungen verbunden ist.

Die OBVSG hat die gesamte Koordination des Projekts, die operativen Schritte sowie die formale ZR-Zuständigkeit für die Normdatei ZDB im OBV übernommen. Sie war für die Planung der Schulungen zuständig, die durch die ZDB Berlin in Wien durchgeführt wurden. Außerdem stellt sie die entsprechende Infrastruktur zur Verfügung. Seit 13.4.2015 ist die neue ZDB-Bearbeitung in Produktion.

Mag. Peter Klien

Die Österreichische Bibliothekenverbund \& Service GmbH (OBVSG) Raimundgasse 1/3, A-1020 Wien

E-Mail: peter.klien@obvsg.at

Website: www.obvsg.at

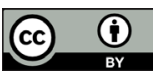

Dieses Werk ist lizenziert unter einer Creative-Commons-Lizenz Namensnennung 4.0 International 